\title{
Filigrane
}

Écoutes psychothérapiques

\section{Métaphores et sexualité : le langage, entre symbole et processus primaire}

\section{Florian Houssier et Clara Duchet}

Volume 20, numéro 1, printemps 2011

La langue vivante de la clinique psychanalytique

URI : https://id.erudit.org/iderudit/1004039ar

DOI : https://doi.org/10.7202/1004039ar

Aller au sommaire du numéro

Éditeur(s)

Revue Santé mentale au Québec

ISSN

1192-1412 (imprimé)

1911-4656 (numérique)

Découvrir la revue

Citer cet article

Houssier, F. \& Duchet, C. (2011). Métaphores et sexualité : le langage, entre symbole et processus primaire. Filigrane, 20(1), 45-54.

https://doi.org/10.7202/1004039ar
Résumé de l'article

Notre propos tend à articuler langage et métaphores, en prenant des exemples dans les champs culturel et psychopathologique. Nous proposons une introduction théorique pour poser quelques points de repères sur les origines de l'accès au langage, avant d'illustrer comment la psychanalyse permet de saisir comment " ça " parle, à la façon d'une vision du monde interne qui se dévoile au fil des mots et de la découverte de leur sens latent ; dans cette perspective, la prégnance des enjeux sexuels ne lasse pas de faire retour, par exemple dans un film. Sur le plan psychopathologique, la répétition de termes précis, appartenant à un champ signifiant commun, peut davantage représenter un point d'achoppement de la problématique d'un patient. 


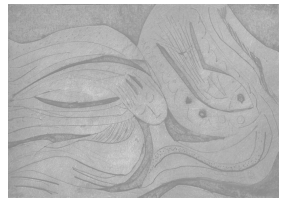

\title{
Métaphores et sexualité: le langage, entre symbole et processus primaire
}

\author{
Florian Houssier et Clara Duchet
}

\begin{abstract}
Notre propos tend à articuler langage et métaphores, en prenant des exemples dans les champs culturel et psychopathologique. Nous proposons une introduction théorique pour poser quelques points de repères sur les origines de l'accès au langage, avant d'illustrer comment la psychanalyse permet de saisir comment "ça " parle, à la façon d'une vision du monde interne qui se dévoile au fil des mots et de la découverte de leur sens latent; dans cette perspective, la prégnance des enjeux sexuels ne lasse pas de faire retour, par exemple dans un film. Sur le plan psychopathologique, la répétition de termes précis, appartenant à un champ signifiant commun, peut davantage représenter un point d'achoppement de la problématique d'un patient.
\end{abstract}

\footnotetext{
S
} igmund Freud (1913) a considéré que la transmission de la culture dans le temps était notamment véhiculée par les différentes formes de la langue, des proverbes aux poèmes en passant par les expressions populaires. Dans l'exploration de cette perspective phylogénétique, il repéra les origines sexuelles du langage. Aujourd'hui, le langage est régulièrement infiltré par des mots d'une autre langue, l'anglais notamment, provoquant parfois des débats passionnés sur la perte supposée d'une culture de la langue, aux détriments d'une langue considérée comme consensuelle et appauvrie. Le linguiste A. Rey (1993) considère pourtant que la langue française s'est rarement mieux portée qu'aujourd'hui, notamment parce qu'elle résulte d'un brassage incomparable, d'un bouillonnement issus de mots provenant du monde entier.

Notre propos tend à articuler langage et métaphores, en prenant des exemples dans les champs culturel et psychopathologique. Une expression telle que «Honni soit qui mal y pense » n'est pas seulement porteuse de signification; elle s'inscrit dans le champ de la sensorialité si on entend la séquence rythmique propre à son énoncé. Sur le plan psychopathologique, la répétition de termes précis, appartenant à un champ signifiant commun, peut davantage représenter un point d'achoppement de la problématique d'un patient, ce que nous développerons à partir des termes récurrents utilisés par Jacques au décours de sa psychothérapie.

Dans un film comme dans l'écoute d'un patient, nous illustrons comment la psychanalyse permet de saisir comment «ça » parle, à la façon d'une vision du monde interne qui se dévoile au fil des mots et de la découverte de leur sens latent; dans 
cette perspective, la prégnance des enjeux sexuels ne lasse pas de faire retour; avant d'illustrer les effets du langage et de la métaphore ou leurs apories, nous proposons un détour théorique pour poser quelques points de repères sur les origines de l'accès au langage.

\section{Investir le langage: d'une illusion nécessaire}

L'enfant ne naît pas seulement dans un bain d'affect, il vient également au monde dans un bain de paroles. Il est d'abord entouré par des adultes qui parlent entre eux, et les échanges corporels avec les adultes, source de plaisir, s'accompagnent de paroles, associées par l'enfant à ces expériences de plaisir.

Semons quelques indices propices à l'investissement libidinal du lien. À la naissance, les bébés reconnaissent la voix de leur mère. Dès la deuxième semaine, la voix humaine déclenche plus fréquemment des sourires que d'autres stimulations sonores. Les bébés d'un mois savent ensuite faire la différence entre des sons aussi proches que «baba» et «papa».

L'échange de regards, l'apparition du sourire, les mimiques ou modifications corporelles sont autant d'évènements que la mère impute à l'usage de sa parole, à travers des chansons, des histoires ou des mots, comme si le bébé en comprenait quelque chose. Et de fait, il en ressent les intonations, la rythmicité, la tonalité des affects accompagnant les énoncés. La mère joue avec les mots, le bébé articulera bientôt usage de mots et jeux divers dans un espace transitionnel partageable (Winnicott, 1975).

L'illusion anticipatrice maternelle transforme ainsi les compétences du bébé en capacités. Si la mère se comporte de manière cohérente, elle fait comme si le psychisme du bébé était déjà organisé. Par exemple, des sons doux stoppent les cris d'un bébé aussi bien qu'une sucette sucrée. En s'adressant à lui comme s'il était un vrai interlocuteur, elle le transforme progressivement en interlocuteur.

Après les premiers vagissements, dans les moments d'éveil calmes et tranquilles, apparaissent les lallations qui sont des bruits articulatoires variés produisant des sons. Leur variété est plus importante que les phonèmes de la langue maternelle, quelle qu'elle soit. Ce qui signifie que lorsque le bébé produit un nombre important de sons, son registre sonore est plus riche que toute autre langue au monde et que l'apprentissage du langage par l'enfant va inclure un désapprentissage d'un certain nombre de possibilités articulatoires. Ce qui caractérise les lallations, c'est leur aspect ludique. Le bébé les répète, comme dans un jeu.

Les adultes ont tendance à reprendre en écho ces lallations mais en modifiant l'intonation. Pour que ces lallations se développent, il est nécessaire que les personnes importantes pour l'enfant les aient écoutées avec plaisir et y ait répondu avec cette manière particulière qui prolonge le désir de l'enfant, bien avant que le message transmis soit compris.

Ces sons répétés font partie des phénomènes transitionnels. Ces lallations continuent pendant toute la période où l'enfant apprend à parler. L'enfant aura plaisir à répéter ces mots, au moment de l'endormissement notamment. 
Ces lallations se prolongeront dans le plaisir qu'obtiendra l'enfant à écouter et répéter des comptines, ou dans le plaisir de la musique et de la poésie. L'accès au langage montre que les premières reconnaissances sont acquises, que les premières différenciations sont en place. L'enfant peut progressivement accepter l'idée que son objet d'amour a une existence propre, que celui-ci existe même lorsque l'enfant ne le perçoit pas. Le langage fait donc partie des premières activités symboliques, contribuant à la maitrise de l'angoisse liée à la perte de l'objet d'amour. Le langage devient alors un outil fondamental pour une nouvelle évolution du fonctionnement psychique. En effet, puisqu'il permet de parler d'objets absents, le langage favorise l'accroissement du monde des représentations internes.

Aussi, à partir du moment où l'enfant peut nommer ses affects, il a à sa disposition un moyen de les élaborer, beaucoup plus efficace que la décharge immédiate de ces affects par la motricité.

Il devient progressivement indispensable pour l'enfant de représenter ce qui ne peut être vu pour lutter contre l'angoisse de l'espace non visible et de la perte de l'objet primordial, la mère. L'enfant peuple cet espace invisible par la projection d'un objet phobogène. Réduire l'angoisse à cet objet permet à l'enfant de la maitriser par sa figuration et par sa limitation spatiale car il est plus facile de trouver un objet contraphobique que lorsque l'angoisse est sans objet défini.

Raconter à l'enfant des contes de fées, même sanglants ou morbides, est une manière de l'aider car les personnages terrifiants ne sont rien d'autre que des figurations d'une angoisse que l'enfant ressent de toute façon. Ces histoires n'augmentent donc pas leur angoisse. La présence de l'adulte, son étayage langagier, lui garantissent un contenant de pensée et une fin heureuse, lui permettant de figurer son angoisse et de jouer avec (Houssier, 2007a).

Il n'en reste pas moins qu'il y a un caractère inassimilable dans le message adulte, lié au sexuel adulte que l'enfant ne peut que refouler ou constituer en crypte. Cet enjeu de transmission dépend donc de la qualité de refoulement et de la métabolisation des fantasmes parentaux; sans cette qualité psychique du refoulement de fantasmes archaïques, l'enfant peut être débordé par l'excitation ou ne peut que rejeter ces contenus qui ne se présentent pas à lui de façon introjectable. Les signifiants énigmatiques (Laplanche, 1987) auraient pour particularité de pouvoir constituer des restes traduisibles psychiquement, sous la forme de représentations; les représentations en attente constituent un potentiel, un stock de représentations potentielles qui vont trouver des points d'ancrage à travers les rencontres du sujet avec son environnement; en revanche, le clivage dans la psychopathologie tend à montrer l'existence de restes intraduisibles ou encore, à travers le symptôme, l'enfant ou l'adolescent traduit ce que les parents n'ont pu intégrer psychiquement, le langage d'un autre, à la façon d'un interprète.

\section{Entre les murs, entre les mots: de quelques métaphores en partage}

Interpréter, c'est également le rôle des acteurs et d'un réalisateur. Le dernier film de L. Cantet, Entre les murs, palme d'or du festival de Cannes en 2008, scrute la 
relation quotidienne professeur-élève dans un crescendo maintenu constamment sous tension.

Ce magnifique film sur le langage éclaire la façon dont les adolescents se l'approprient en le transformant. Certains adolescents auraient d'ailleurs délaissé l'américanisation de la langue française pour adopter une expression nouvelle: au lieu d'affirmer: "Je suis cool», ils disent actuellement: "Je suis moelleux ». Cette créativité maintenant la langue en mouvement se retrouve dans le film lorsque deux adolescentes, sans doute un peu trop séduites par leur professeur maître ès provocation, lui disent en substance: "Faut pas charrier». Or, c'est bien ce qui infiltre tout le film, cette circulation de la libido (sa viscosité à l'œuvre, aussi) qui rend le lien si complexe, si proche et si violent à la fois au moment de l'adolescence. Charrier n'évoque pas seulement la charrette et ses transports ici émotionnels, mais aussi, en suivant le fil de sa définition: l'exagération, l'application d'une loi étrangère à toute raison.

Qu'a bien pu dire le professeur pour s'entendre dire qu'il charrie? Quel déplacement - toujours les transports - a encore frappé? D'une expression à l'autre, il faut se remettre à entendre. Nous sommes au début du film, les enjeux sont encore balbutiants, l'année commence, c'est la mise en place. L'enseignant est au travail, avec une belle énergie, celle de l'espoir. À propos du sens d'un mot, les élèves butent; il insiste, veut les attirer dans la logique du sens en s'adressant à tous, les considérant comme doués d'une intelligence précise pour trouver ce qui échappe. Il lance enfin: «Est-ce que ça ne vous met pas la puce à l'oreille?»

Arrêt sur expression. Qu'est-il en train de dire? Il veut certes attirer leur attention, les conduire comme tout pédagogue à un point donné d'intelligence éclairée. Mais lorsqu'on s'intéresse à l'origine de cette expression, on comprend autre chose, ailleurs. Si le sens de l'expression renvoie bien à l'idée de se douter de quelque chose ou se méfier de quelqu'un, un retour aux origines nous indique qu'au treizième siècle, cette expression symbolisait le désir sexuel qu'on peut ressentir pour une personne. Et, lorsqu'un amant trouvait une puce sur le corps de sa maîtresse, il lui arrivait de la placer dans un médaillon, d'où la coutume d'alors, mettre une puce en guise de boucle d'oreille (Rey et Chantereau, 1993).

Quatre siècles plus tard, le sens se modifia: les puces ayant proliféré dans toutes les couches de la société, celui qui souffrait de démangeaisons pouvait avoir un air inquiétant. À partir de la gestuelle, l'expression devint alors synonyme d'être inquiet, agité, voir fou. Puis, de fil en aiguille, tricotage indispensable, le sens s'est modifié pour glisser jusqu'à celui d'aujourd'hui, issu de l'idée de se douter de quelque chose.

Retour sur image: en tentant de tisser sa toile de savoir, l'enseignant traduit un désir de séduction à double tranchant; les mots charriant ses pulsions le débordent lorsqu'il traite deux adolescentes de « pétasses ». Or, sur ce sujet, elles en connaissent un rayon et le mettent devant la réalité des mots: une pétasse est une putain, cela et rien d'autre, quelque soit les protestations peu convaincantes que le professeur tente de donner, comme pour se camoufler. Un camouflet, c'est ce qu'il recevra à plus d'un titre. Voici un des enjeux véhiculés par le langage, qui se révèle: les enjeux relationnels 
prennent le pas sur l'apprentissage, et la relation transférentielle est bien au cour de toute «éducation au savoir» (Houssier, 2010).

\section{Expressions dans la psychothérapie: symbolisation ou émergences en processus primaires?}

Nous avons déjà eu l'occasion de proposer l'idée selon laquelle l'usage de métaphores, ici dans le contexte de la psychothérapie d'un patient souffrant d'une problématique pédophile, sont autant de révélateurs de la prégnance de traumatismes psycho-corporels précoces (Houssier, 2008). Ce n'est pas l'angle que nous choisissons ici, mais plutôt celui d'une interrogation de la valeur du langage. L'usage récurrent de métaphores thermiques dans les énoncés de Jacques interroge la qualité de ces métaphores: révélateurs d'une souffrance figée, ou énonciation symbolique de traumatismes qui ne peuvent se dire autrement?

\section{Articulation entre traumatismes infantiles et métaphores thermiques}

Agé de quarante ans, Jacques engage de sa propre initiative une démarche psychothérapique en libéral, parallèle à son suivi socio-judiciaire. Sa demande repose sur le constat d'une impasse concernant ses désirs sexuels pédophiles, constat accompagné du projet de trouver une issue moins destructrice à sa sexualité. «Je ne sais pas aimer ", nous dit-il. Derrière cette demande manifeste, se révèle progressivement le poids d'expériences traumatiques à la fois transgénérationnelles et intersubjectives.

Nous le recevons à raison d'une fois par semaine en face à face, à la suite de sa seconde condamnation pour des faits de pédophilie. Jacques a récemment pris conscience qu'il ne se sentait pas appartenir à la communauté des humains. Ainsi, il se sent vivre dans un monde personnel où rien ne compte d'autre que ses désirs passionnels, sur fond de relations explosives avec sa mère. Celle-ci apparait comme une figure incontournable de sa vie, comme en constante surimpression - et non en toile de fond - de sa vie psychique. Son adolescence est marquée par l'excitation sexuelle qu'il ressent pour elle, excitation qu'il qualifie de "monstrueuse ", à l'origine d'une érection qui le remplit d'horreur et de gêne. Enfant, il a rêvé d'elle se transformant en monstre.

Il relate un souvenir, celui d'une baby-sitter adolescente qui, alors qu'il a cinq ans, le force à mettre ses mains sur des plaques de cuisson brûlantes. Comme à l'annonce de la mort de son père, il ne ressent rien sur le moment, la douleur étant avant tout liée non à son corps et à sa peau, mais au sentiment d'avoir été abusé.

Cette dimension brûlante de la relation aux femmes dans les générations constitue une composante essentielle de sa vie fantasmatique. Les scènes incestueuses passées se déplient maintenant au fil des séances; sa mère, pour dire qu'elle veut qu'il fonde une famille, lui dit: "Je veux que tu me fasses un enfant»; cette proposition, ou en tout cas la façon dont Jacques la reçoit, résonne avec le fait que, de neuf à quatorze ans, entre le départ de son père et l'arrivée de son beau-père, il a dormi dans le lit de sa mère. Lors de son premier passage à l'acte pédophile, Jacques séduit un enfant pour l'amener chez sa mère, chez qui il vit encore à ce moment-là. C'est là une façon 
de réaliser le désir de sa mère, «lui faire un enfant», expression qu'il a entendu de façon littérale. L'interprétation de cette demande a simultanément pour effet d'annuler la différence des générations, Jacques n'étant plus en position d'être l'enfant de sa mère, mais celui qui peut lui en donner un. Il se sent ainsi annulé en tant que sujet qui peut se repérer dans le temps et dans la communauté des hommes grâce à la différence des générations et l'interdit qui la structure. Le risque d'enfermement dans une bulle narcissique, passant par un fantasme d'auto-engendrement et de perte de toute asymétrie dans le lien à autrui, participe du défaut potentiel de construction de la capacité à devenir sujet et à s'ouvrir à un autre différencié. Le travail psychique d'individuation s'organise à partir de ces conflits en mouvements constants portant sur les trois différences fondatrices du processus de subjectivation - différence soiautre, des sexes et des générations (Houssier, 2005).

Pour Jacques, toute relation aux adultes, sur le modèle de celle à sa mère, est brûlante; de ce fait, par peur d'être brûlé, il s'organise défensivement pour que personne n'effracte sa sphère d'intimité: il veut donc rester «intouchable» et ne laisse personne entrer en lui, par exemple en partageant son intimité dans une relation de couple. Dans la série des scènes brûlantes, il évoque une mère colérique qui, enfant, lui donnait de terribles fessées qu'il vivait comme violentes et interminables, notamment lorsqu'il ramenait de mauvaises notes. "Ma mère me terrorisait », dit-il pour expliquer sa réussite professionnelle actuelle. Enfant, le lien à la mère est scandé par des phases où règnent l'indifférenciation et la terreur, et par d'autres phases de discontinuité du lien marquées par le caractère imprévisible de la relation à la mère, éruptive «comme un volcan».

Se sentir comme un objet passivé et sous emprise, il s'en défend dans son domaine professionnel par une attitude autoritaire et colérique. «Ca me fait flamber, les relations de soumission ", dit-il; il se met en colère avec ses collègues pour soumettre l'autre par peur d'être soumis. Il associe sur sa mère en évoquant que lorsque l'agressivité monte entre elle et lui, «c'est le feu». Mais en parlant de ces relations, il se rend compte de l'effet effrayant qu'il peut produire sur les autres, et qu'il ne veut plus utiliser. Lorsqu'il envisage de mettre de la distance entre sa mère et lui, relation dont il considère qu'elle a encore trop d'impact sur lui, il «prend la température » et se rend compte que sa mère accepte sans sourciller, ce qui le déçoit car il s'attendait à « un feu d'artifice».

Parmi les premières formalisations de l'interdit du toucher (Anzieu, 1985), au service de l'auto-conservation, est énoncé : «Tu ne mettras pas ta main sur le feu », injonction qu'on retrouve dans certains contes pour enfants (Houssier, 2007b); ici, elle aurait été mise en acte de façon retournée, dans un lien traumatique d'emprise lors de la scène impliquant la baby-sitter. Cette construction secondarisée participe à la représentation des traces de traumatismes précoces passant par la peau et les brûlures; elle confère une place centrale à la destructivité dans le lien. L'excitation déliée se retourne en scénario pervers dans la séduction pédophile, où Jacques amène l'enfant incestueux à sa mère, séduction mêlant une parole supposée bonne à un acte cruel, un cadeau empoisonné. 


\section{Une jonction entre sens et représentation: pour qui ?}

Les extraits choisis de la psychothérapie de Jacques restituent l'importance des traumatismes sexuels et sensoriels dans la psychogenèse de la pédophilie, touchant notamment les enveloppes visuelles, sonores et tactiles. Pourtant, les métaphores concernant la peau et les brûlures émergent de façon sans doute plus décisive dans les déterminants psychiques de la problématique de Jacques. À travers les métaphores thermiques utilisées par Jacques, c'est la fonction même du langage qui est interrogée.

La persistance des images traumatiques ponctuées de métaphores brûlantes chez Jacques constituent autant de témoignages d'un lien trop chaud pour ne pas faire effraction et laisser des traces indélébiles, collapsant précocement peaux physique et psychique. Si tout humain pense avec sa peau, le récit de Jacques interroge : est-ce avec sa peau ou celle d'une autre qu'il pense, à partir d'une peau commune créant un espace insécable?

Le froid et le chaud, équivalents d'enveloppes thermiques, représentent aussi les extrêmes: l'impuissance et la toute-puissance, le tout ou rien, l'indifférence ou l'expression pulsionnelle brute.

Il a le souvenir de voix d'hommes calme, ce qu'il dit aussi nous concernant; il envie cette capacité à refroidir les sentiments sans les désaffectiver. La bienveillance partageable s'oppose à l'indifférence et l'indifférenciation soi-autrui, la sonorité de la voix d'homme possédant alors une fonction tierce dans le sens du refroidissement des excitations débordantes. L'enjeu pour Jacques est de pouvoir passer de l'expérience d'une enveloppe de chaleur brûlante qui troue la possibilité d'une enveloppe pare-excitante - c'est trop chaud, ou encore « $j$ 'ai un retour de flammes» - à des représentations de bases ouvrant sur l'articulation des sensations avec des pensées correspondantes. Lorsqu'il évoque la voix d'un homme calme, Jacques envie la possibilité de refroidir les affects dans le sens de l'usage d'un pare-feu (ou excitation). Ce pare-feu permet de mélanger le chaud de la passion érotique d'emprise au refroidissement des processus secondaires à visée élaborative.

Si on considère que le corps est le lieu où convergent les effets du sens donné aux mots et du traumatisme (Vilela, 2004), les mots jouent un rôle de jonction : les associations verbales permettent à la pensée d'investir les traces mnésiques traumatiques en suspens, dépliées dans le langage métaphorique repris et traduit par le psychanalyste. «Ma peau est brûlée» fait partie des exemples d'articulation entre la charge quantitative de l'affect et sa représentation, selon J. Kristeva (1996). Creusant ce sillon, cet auteur considère que le langage rend possible le passage du signifié abstrait au traumatisme inconscient et même corporel. Les métaphores thermiques qui tapissent le monde interne de Jacques sont donc des traces de souvenirs reconvoquées par le lien transférentiel et le dispositif mis en place. Reprenant le travail de S. Freud (1895), J. Kristeva $(1996,56)$ interroge «l'équivalence entre pensée et perception, et notamment leur nœud originaire qu'est l'hallucination ». On peut faire l'hypothèse que les métaphores de Jacques conservent une teneur et un quantum hallucinatoires dès lors qu'il ne s'entend pas parler, sans la capacité de réflexivité qu'il développe progressivement en entendant et commentant les interventions du psychanalyste. Ainsi, 
lorsqu'il s'entend dire, au décours d'un énoncé, « [...] depuis que je suis devenu une femme », il comprend immédiatement ce qui vient de lui échapper, en étant très surpris de ce qu'il se fait entendre à lui-même. Être devenu une femme perd alors sa charge hallucinatoire pour s'insérer dans les contenus secondarisés de la séance. Il illustre aussi sa capacité réflexive en disant spontanément: «Depuis que je viens vous voir, je me parle à moi-même».

Pour autant, Jacques s'entend-il parler, s'écoute-t-il ? Au fur et à mesure, il repère la dimension répétitive de ses métaphores brûlantes, initialement pointée par l'analyste. En reprenant la métaphore de la perle formée par le grain de sable développée par Janin (1996), nous considérons que la métaphore parle avant tout à l'analyste, le contre-transfert précédant le transfert. Dans cette construction théorique, le grain de sable vient symboliser l'événement (ou sa trace traumatique) à partir duquel «les fantasmes vont se développer comme les concrétions perlières le font autour du grain de sable réel» (Janin, 1996, 21). Pour ce patient, les grains de sable ne sont pas insignifiants pour lui (en témoignent ses capacités d'association sur ses événements traumatiques), mais pour qu'ils deviennent tout à fait perles c'est-à-dire symbolisables, il devra supporter de se décoller de la peau maternelle et par là même supporter de s'écouter parler en donnant valeur et pouvoir au langage des hommes plutôt qu'à leurs actes. Le psychanalyste, dans sa position de tiers, est là pour l'y aider... Pendant ce temps, les métaphores thermiques travaillent du côté de l'analyste en venant confirmer l'hypothèse d'une révélation, par le langage métaphorique, de la prégnance des traumatismes psycho-corporels précoces chez Jacques.

Approfondir les aspects thérapeutiques et transférentiels en œuvre avec ce type de patient ouvre sur la compréhension de la qualité psychique propre à la métaphore; celle-ci peut graduellement s'inscrire dans des processus de transformation psychique au service de cette relation à un autre. C'est parce que les métaphores de Jacques font sens pour le psychanalyste que progressivement, par effet miroir, «ça » fait sens pour lui. L'énoncé langagier possède un après-coup, dans la possibilité de la saisie de son sens, ou d'un sens caché. Sa façon d'éprouver ce que les mots provoquent en lui, permet la différenciation avec l'interlocuteur: "Ce que vous me dites là, je sens que ça me serre à l'intérieur », dit-il en montrant sa poitrine.

\section{Conclusion}

Il n'est pas question ici de donner une réponse, ni d'analyser ce cas en fonction d'objectifs thérapeutiques, mais plutôt de comprendre comment le langage s'insère dans la dynamique transférentielle. Les mots ne sont pas seulement des viatiques pour symboliser et faire passer idées et affects; ils sont aussi le lieu du malentendu, où se glisse l'histoire du patient et des effets de la rencontre intersubjective. Le contexte clinique d'émergence des métaphores thermiques n'est pas sans évoquer la proximité des maladies psychosomatiques et transgressives quant à l'indiscrimination entre les affects et les représentations (Green, 1980). Le travail thérapeutique passe par la reconnaissance verbale, véhicule des affects du psychanalyste, de ce qu'a pu vivre l'agresseur au moment de son acte, en tant que réveil de traces de trauma- 
tismes autrefois subis. L'usage des mots, sans garantir une quelconque certitude thérapeutique, ouvre une voie praticable pour permettre la reconstruction de processus psychiques afin d'accéder à un sentiment d'identité continu et soutenu par des représentations supportables; ce passage aux mots inclut la participation active du psychanalyste, comparable à celle utilisée par les psychosomaticiens ou par un pionnier comme A. Aichhorn dans sa pratique avec les jeunes délinquants (Houssier, Marty, 2007).

Les interventions dépassionnées de l'analyste, accompagnées de séquences verbales à la chaleur tempérée, peuvent également témoigner de son expérience de la condition humaine. Ces messages verbaux, leur rythmicité et leur ton, tendent à restaurer un contact psychisé peau à peau chargé de sens qui réintègre le sujet dans la communauté des humains, l'extirpant d'une passion déshumanisante qui, dans le cas de Jacques, l'isole dans une mixion brûlante entre érotisme narcissique et destructivité.

Si la psychanalyse est aujourd'hui contestée, elle semble pouvoir résister aux secousses qui la traversent en mettant les mots et le transfert au centre d'un lien unique; sa popularité reste intacte, certains termes étant passés dans le langage commun, au risque de l'évidement de son contenu initial. Sa reconnaissance - qui n'a pas besoin de parler et d'être entendu-reconnu? - passe par son aspect transculturel; l'usage du langage par le psychanalyste transcende les différences pour tenter, entre malentendus et interprétations éclairantes, d'y voir plus clair.

Florian Houssier, Institut de psychologie Laboratoire de psychologie - Clinique et de Psychopathologie (LPCP) - E. A. 4056 Université Paris Descartes Florian.Houssier@parisdescartes.fr

Clara Duchet, Institut de psychologie Laboratoire de psychologie - Clinique et de Psychopathologie (LPCP) - E. A. 4056

Université Paris Descartes Clara.Duchet@parisdescartes.fr

\section{Références}

ANZIEU, D., 1985, Le Moi-peau, Bordas, Paris.

FREUD, S., 1956, Esquisse d'une psychologie scientifique (1895), La naissance de la psychanalyse, Paris, PUF., 1956, 307-396.

FREUD, S., 1913, Totem et tabou, Payot, Paris, 1947.

GREEN, A., 1980, Passions et destins des passions, La folie privée, 1990, Gallimard, Paris, 141-196.

HOUSSIER, F., 2005, L'enfant jumeau et son devenir. Indifférenciation et subjectivation dans le lien sororal, Topique, 93, 91-103. 
HOUSSIER, F., 2007a, Mythe phylogénétique, rêve et conte pour enfant : la permanence d'une trace infanticide dans la culture freudienne, Le Divan familial, 19, 131-140.

HOUSSIER, F., 2007b, Pierre l'Ebouriffé et les souhaits infanticides : du conte à la clinique de la parentalité, Psychiatrie de l'enfant, 1, 2, 483-502.

HOUSSIER, F., 2008, Inceste, pédophilie et déni d'existence, Le Divan familial, 20, 171-184.

HOUSSIER, F., 2010, L'école d'Anna Freud. Créativité et controverses, Éditions Campagne Première, Paris. HOUSSIER, F., MARTY, F., 2007, August Aichhorn, Cliniques de la délinquance, Champ Social, Nîmes. JANIN, C., 1996, Figures et destins du traumatisme, PUF, Paris.

KRISTEVA, J., 1996, Sens et non-sens de la révolte. Pouvoirs et limites de la psychanalyse, 1, Fayard, Paris. LAPLANCHE, J., 1987, Nouveaux fondements pour la psychanalyse, PUF, Paris.

REY, A., 1993, Préface, in Rey, A., Chantreau, S., éds., Dictionnaire des expressions et locutions, Le Robert, non numéroté, Paris.

REY, A., CHANTREAU, S., 1993, Dictionnaire des expressions et locutions, Le Robert, Paris.

VILELA, I., 2004, Cures de paroles : entre représentations de mots et représentations de choses, Marges linguistiques, 8, 139-152.

WINNICOTT, D. W., 1975, Jeu et réalité, Gallimard, Paris. 